lich inhalierten Budesonid-Dosis an. Dies kann als Hinweis auf eine verminderte Deposition von Proteoglykanen in den bronchialen Basalmembranen infolge der Glukokortikoidgabe gewertet werden. Ein Unterschied in der Glykosaminoglykan-Ausscheidung zwischen ato- pischen und nicht atopischen Probanden war nicht festzustellen.

Fazit: Die Ausscheidung von Glycosaminoglykanen im Urin steigt bei Kindern mit Asthma unter Behandlung mit inhalativen Glukokortikoiden an. Dies kann als Hinweis auf einen Anti-Remodeling-Effekt interpretiert werden. $\quad b k$

Priftis KN et al. Increased glycosaminoglycans in the urine of asthmatic children on inhaled corticosteroids. Pediatr Allergy Immunol 2006; 17: 194-8

\title{
Prävalenzanstieg ohne klaren Trend
}

\section{Im Rahmen der International Study of Asthma and Allergies in Childhood (ISAAC) wurden erstmals von 1992 bis 1997 weltweit Prävalenzdaten allergischer Erkrankungen erhoben. Mit den gleichen Prüfinstrumenten erfolgte nun in der ISAAC-Phase drei eine erneute Erhebung.}

F ür die Phase drei von ISAAC wurden in den Jahren 2002 und 2003 193.404 Kinder zwischen sechs und sieben Jahren in 66 Zentren in 37 Ländern anhand eines standardisierten Fragebogens zum Vorliegen von Asthma, allergischer Rhinokonjunktivitis und Ekzemen befragt. Dasselbe galt für 304.679

\begin{abstract}
Was ist eigentlich ISAAC?
Anfang der goer Jahre des letzten Jahrhunderts versuchten zwei Forschergruppen, eine aus Bochum und eine aus Auckland, Neuseeland, multinationale Daten zur Prävalenz allergischer Erkrankungen zu erheben. Die Fusion beider Initiativen im Jahr 1991 war die Geburtsstunde der International Study of Asthma and Allergies in Childhood (ISAAC). In der Phase eins wurden in den Jahren 1992 bis 1997 über 700.000 Kinder aus 56 Staaten zum Vorliegen von Asthma bronchiale, allergischer Rhinitis und atopischem Ekzem befragt. Es folgte von 1998 bis 2004 die Phase zwei, in der in ausgewählten Ländern verschiedene epidemiologische Fragestellungen vertieft wurden Die aktuelle Phase drei schließlich stellt mit geringen Abweichungen eine $\mathrm{Neu}$ auflage der Datenerhebung aus Phase eins dar und erlaubt somit vor allem die Darstellung der zeitlichen Veränderung der ermittelten regionalen Prävalenzdaten. Das große Verdienst von ISAAC ist neben der Datenerhebung die Entwicklung eines standardisierten Fragebogens als valides Instrument zur Ermittlung von Prävalenzdaten. Weitere Informationen bietet unter http://isaac.auckland.ac.nz die ISAAC-Website.
\end{abstract}

Kinder zwischen 13 und 14 Jahren in 106 Zentren in 56 Ländern. Die Probanden wurden jeweils als zufällige Stichproben von Schulen in einer vorher definierten Gegend des jeweiligen Landes rekrutiert.

Die Phase drei der ISAAC-Studie konnte durchschnittlich sieben Jahre nach der Phase eins abgeschlossen werden. In den meisten Zentren ergab sich eine signifikante Prävalenzänderung für mindestens eine der abgefragten drei atopischen Erkrankungen. Die Veränderungen waren am ausgeprägtesten für Ekzeme bei den Jüngeren und für allergische Rhinokonjunktivitis in beiden Altersgruppen. Ein Prävalenzanstieg aller drei atopischen Erkrankungen in beiden Altersgruppen wurde vor allem in den Staaten der asiatischen Pazifikküste und in Indien registriert.

Insgesamt waren Prävalenzzunahmen zweimal so häufig wie Prävalenzrückgänge, wobei die Anstiege vor allem in der Gruppe der sechs- bis siebenjährigen Kinder auftraten. Eine Ausnahme war die Asthmasymptomatik in der Gruppe der 13- bis 14-Jährigen, bei der in Gebieten mit einer hohen Prävalenz in Phase eins jetzt ein Rückgang zu verzeichnen war.

Fazit: Sieben Jahre nach Abschluss von ISAAC-Phase eins wurden jetzt in einer wiederholten Erhebung erneut Prävalenzdaten zu Asthma, Rhinokonjunktivitis und Ekzem bei Kindern erhoben. Es ergab sich vor allem bei den Jüngeren
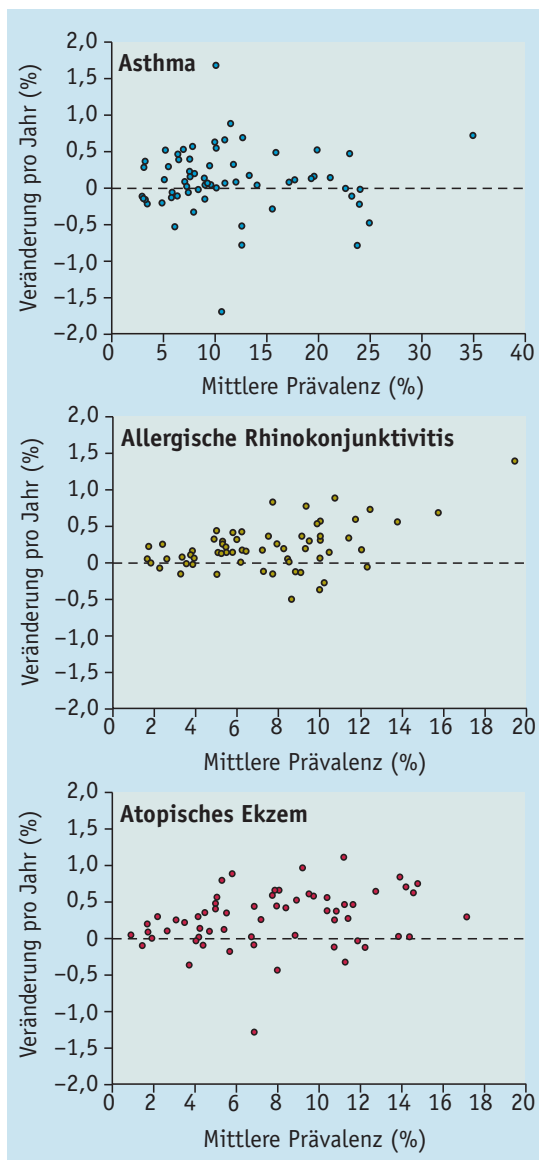

Veränderung der Prävalenz von Asthma bronchiale, allergischer Rhinokonjunktivitis und atopischem Ekzem bei sechsbis siebenjährigen Kindern im Vergleich der ISAAC-Phasen eins und drei. Jeder Punkt repräsentiert ein Land.

eine Zunahme der Allergieprävalenz, in den meisten Zentren waren die Veränderungen aber komplex und ohne einheitlichen Trend.

$b k$

Asher Ml et al. Worldwide time trends in the prevalence of symptoms of asthma, allergic rhinoconjunctivitis, and eczema in childhood: ISAAC Phases One and Three repeat multicountry cross-sectional surveys. Lancet 2006; 368: 733-43 